\section{Research Square}

Preprints are preliminary reports that have not undergone peer review.

They should not be considered conclusive, used to inform clinical practice, or referenced by the media as validated information.

\title{
Tenascin-C Promotes Skin Inflammation and Wounds Healing after Scalding in Rats by Inducing Infiltration of Macrophages
}

\section{Weiquan Zhong}

Southern Medical University Nanfang Hospital

Jin Wang

Southern Medical University Nanfang Hospital

Wenjun Liu

The Third Affiliated Hospital of Guangzhou Medical University

\section{Yixin Mao}

Southern Medical University Nanfang Hospital

Hao Liu ( $\nabla$ specificgz@163.com )

Southern Medical University Nanfang Hospital https://orcid.org/0000-0002-9306-7663

Kai Qian

Southern Medical University Nanfang Hospital

\section{Research Article}

Keywords: Tenascin-C(TNC), macrophage, deep second-degree scald, inflammation reaction, wound healing, inflamm-aging

Posted Date: February 17th, 2022

DOI: https://doi.org/10.21203/rs.3.rs-1339783/v1

License: (c) (i) This work is licensed under a Creative Commons Attribution 4.0 International License. Read Full License 


\section{Abstract}

We have known that Tenascin-C(TNC) has high expression in chronic inflammation. This research explores the proinflammatory mechanism and further discusses the relationship between the TNC and the macrophages in the inflammatory response. First, we successfully built the deep second-degree scald models of rats. Then we found that the level of TNC increased rapidly in the skin of young rats just after scalding, and its tendency also stays increased within a week in old rats. We also observed the infiltration of inflammatory cells in the scalded skin of rats by Immunohistochemistry (IHC) staining. In addition, we found that tenascin-c could confer a proangiogenic effect to macrophages. By monitoring the level of VEGF, CCL2, and CCR2, we can find out the relationship between TNC and macrophages. We concluded that TNC could promote skin inflammation and wound healing in rats after scalding by inducing infiltration of macrophages.

\section{Introduction}

TNC is a hexameric, polymorphic extracellular matrix glycoprotein. The molecule has four different domains, each of which can bind to cell surface protein receptors. ${ }^{[1]}$ TNC has high expression during embryonic development, tissue

repair and in pathological situations such as chronic inflammation and cancer. ${ }^{[2]}$ Although TNC has limited expression in healthy tissues, it is rapidly up-regulating after tissue injury. Naturally, its expression is short-lived. When tissue repair is completing, its mRNA level will be down-regulated, and protein expression will also decrease. ${ }^{[3]}$

Macrophages are the primary regulator cells of inflammation. It has various functions in injury, including promoting and eliminating inflammation, angiogenesis, and wound healing. ${ }^{\left[{ }^{[9]}\right.}$ Macrophages are classified into M1 classically activated macrophages and M2 alternatively activated macrophages. M1 macrophages are produced in an inflammatory environment dominated by TLR signals ${ }^{[13]}$, and activated M1 macrophages can produce many proinflammatory mediators and cytokines. ${ }^{[14]}$ Macrophages activated by anti-inflammatory cytokines are called M2 macrophages. They exist in the environment of CD4 + Th2 responses, such as inhibiting inflammation, promoting tissue remodeling, and angiogenesis. [15]

It currently knows that TNC is highly expressed in inflammation and wound healing. Tenascin-C can also impact on cell proliferation through activation of the epidermal growth factor (EGF)-receptor and inflammatory signaling pathways ${ }^{[1]}$. Macrophages are the primary cells of the inflammatory response and interact with many proinflammatory mediators. However, tenascin-C appears to exhibit a cell-type specific effect on migration; it inhibits monocyte chemotaxis in vitro and tenascin-C-null mice exhibit increased migration of monocytes and macro-phages in mammary tumor stroma ${ }^{[4]}$. Through this experiment, we established the scalded skin rat model to explore whether TNC is related to macrophage infiltration to promote inflammation and wound healing. 


\section{Materials And Methods}

\section{Animals}

In this study, we used male SD rats. Among them, young rats are eight weeks old and weigh about 250g; old rats are 16 months old and weigh about $550 \mathrm{~g}$. We performed all operations under pentobarbital sodium anesthesia. The procedures for care and use of animals were approved by the Ethics Committee of the Southern Medical University and all applicable institutional and governmental regulations concerning the ethical use of animals were followed.

\section{Establishment of rat scald skin model}

We anesthetized male rats by intraperitoneal injection of $3 \%$ pentobarbital sodium according to 1.7 $\mathrm{mL} / \mathrm{kg}$, exposed the skins, and used a constant temperature and pressure scald instrument at $94^{\circ} \mathrm{C}$ for 8 seconds with the pressure of $500 \mathrm{~g}$ to cause deep second-degree scald on the back of the rats. The back

of each rat had the same wound surface $(1 \mathrm{~cm} \times 3 \mathrm{~cm})$, and we gave the rats an intraperitoneal injection of $5 \mathrm{~mL}$ saline to prevent shock. Subsequently, we divided the rats into young and old groups, divided into the control group and the $1 \mathrm{st}$ day, 4th day, and 7th day groups after the scalding.All animals were kept in a pathogen-free environment and fed with national standard rodent feed. To avoid other drugs from affecting wound healing, we only use sterile saline gauze wet compress. We raised three rats in each group. To observe and record the scab formation, scab removal, and healing time of the rat wound.

\section{Enzyme-Linked Immunosorbent Assay (ELISA)}

Before scald and 1st day, 4th day, and 7th day after scald, blood was taken from the eye socket, placed at room temperature for 20 minutes, centrifuged at 3000r/min for 15 minutes, and collected the liquid supernatant. Firstly, we diluted the antigen to an appropriate concentration with the coating diluent, labeled the reaction wells with a blocking enzyme, and removed the bubbles in each well. Then establish a suitable concentration gradient to add the sample to be tested and the enzyme-labeled antibody. Finally, add $100 \mu \mathrm{l}$ of TMB-hydrogen peroxide urea solution to each well, place it at $37^{\circ} \mathrm{C}$ in the dark for $3-5$ minutes, and add stop solution to develop color. The expression levels of rat serum TNC, VEGF, and CCL2 were determined using the ELISA method.

\section{Quantitative Real-time PCR(RT-qPCR)}

We took the normal skin tissues and scald skin tissues of the control group, 1st day, 4th day, 7th day groups of young rats and old rats. First, we used TRIzol one-step method to extract RNA from rat scalded tissues and dried RNA at room temperature. Then add an appropriate amount of RNose-free solution to the sample to dissolve the precipitate. Follow the instructions of TaKaRa for reverse transcription. In the fourth step, after preparing the qPCR reaction system, we put the sample on the qPCR reaction machine for the reaction. Finally, we calculated the gene expression with a CT value. The mRNA expression levels of TNC, VEGF, and CCR2 in skin tissues were detected using RT-qPCR.

\section{Western blot}


We took the normal skin tissues and scald skin tissues of the control group, 1st day, 4th day, 7th day groups of young rats and old rats. Then we used western blot to detect the protein expression level of TNC, VEGF, and CCR2. Firstly the tissue protein samples were transferred to a polyvinylidene fluoride membrane after SDS-PAGE, blocked with $5 \%$ skimmed milk powder at $37^{\circ} \mathrm{C}$ for one hour, washed with TBST buffer three times, 5 min each time, and then added l-antibody and incubated overnight at $4^{\circ} \mathrm{C}$. The next day, anti-ll was added and incubated at $37^{\circ} \mathrm{C}$ for one hour and then developed with enhanced chemiluminescence $(E C L)$ reagent.

\section{Immunohistochemistry (IHC) staining}

The normal skin tissues and scald skin tissues of the control group, 1st day, 4th day, 7th day groups of young rats and old rats were taken and fix them with formalin. Then the rat tissues were dehydrated, embedded in wax blocks, sectioned, and processed on glass slides. After the slides rinsing with distilled water, we added EDTA and sodium citrate antigen retrieval solution. Then the slides were rinsed with PBS buffer, $3 \%$ hydrogen peroxide solution was added to the block for 10 minutes, and then stained. IHC detected the protein expression of CD68 to observe the infiltration of macrophages.

\section{Hematoxylin-eosin(HE) staining}

To take the normal skin tissues and scald skin tissues of the control group, 1st day, 4th day, 7th day groups of young rats and old rats, and fix them in $4 \%$ formaldehyde solution for pathological preparation. Then the rat tissues were dehydrated, embedded in wax blocks, sectioned, and processed on glass slides. After rinsing the slides with distilled water, they were stained with hematoxylin for 3 minutes and then differentiated with hydrochloric acid and alcohol. Then rinse the slide with distilled water again and add eosin staining for 20 seconds. Finally, the slides are dehydrated and mounted with gum. To observe the pathological would changes, including inflammation and its strength by HE.

\section{Statistical analysis}

All data were analyzed using SPSS 20 software. We performed the comparison of means between two groups by using a t-test. Furthermore, we used a single-factor analysis of variance to perform the comparison between multiple groups. ( $\mathrm{P} \leq 0.05$ asset as statistically significant)

The ELISA test showed that compared with the control group, the serum TNC concentration after scalding of young rats increased firstly and then decreased, while the TNC concentration of old rats decreased firstly and then slightly increased (A). The serum VEGF concentration of young rats increased after scalding and then decreased, while the serum VEGF concentration of old rats did not change significantly (B). The serum CCL2 concentration of young rats increased significantly after scalding, while the serum CCL2 concentration of old rats increased and then decreased to no significant difference from the control group (C). ( ${ }^{*}$ compared with the control group, $\mathrm{P}<0.05$; \#compared with the scalded 1 st day group, $\mathrm{P}<$ 0.05). (Fig. 2)

RT-qPCR detection showed that compared with the control group, the expression of TNC mRNA in young rats' skin after scalding decreased first and then increased significantly, while the expression of TNC 
mRNA in the skin of old rats decreased (D). The expression of VEGF mRNA in the skin of young rats after scald significantly reduced immediately, while the expression of VEGF mRNA in old rats significantly reduced on the 4th day after scalding (E). The expression of CCR2 mRNA in young rats increased firstly and then decreased after scalding, while CCR2 mRNA in old rats increased and then decreased, and then increased again on the 7th day $(\mathrm{F})$. ( ${ }^{*}$ compared with the control group, $\mathrm{P}<0.05$; \#compared with the scalded 1st day, $\mathrm{P}<0.05)($ Fig. 3 )

\section{Results}

In this experiment, a scald instrument was used to steam scald rats to construct a rat scald model to exclude the influence of gravity, pressure, and other factors. When the scalding temperature is the same, the scald time becomes the only factor that controls the depth of the scald. Through this model construction method, we successfully constructed multiple groups of rat scald models.

TNC participates in the inflammatory response and promotes tissue repair.

We measured the concentration of TNC in the serum with an ELISA kit method and found that the TNC concentration in young rats increased significantly on the first day after scalding and then gradually decreased. It further proves that TNC is expressing rapidly when tissue is injured, but tissues slowly drop after repair. (Fig. 2) Similarly, we used RT-qPCR to measure the expression of TNCmRNA and found that although the expression of TNCmRNA in the skin tissue of young rats on the first day after scalding decreased compared with the control group, the expression of TNCmRNA in one week Still an upward trend. (Fig. 3)

The expression of VEGFA and CCR2 is directly proportional to the expression of TNC.

We used Western blot to detect the expression of the target protein in the scalded skin tissue of rats. We selected $\beta$-Actin as the internal reference index of Western blot. When it is continuously and stably expressing, we compare the expressions of TNC, VEGFA, and CCR2 proteins to calculate their relative expression levels. It can found that the expression of VEGFA and CCR2 is directly proportional to the expression of TNC. This result indicates that inflammation occurs in the skin tissue of rats after scalding, and the increased VEGF factors in the inflammation site can promote angiogenesis; at the same time, CCR2 is necessary for the recruitment of macrophages in the inflammation site. In summary, skin inflammation after scalding will cause an increase in TNC content, macrophages infiltration, and angiogenesis. (Fig. 4)

Macrophages infiltrate the inflammatory response.

We measured by RT-qPCR that the relative expression of CCR2mRNA in both young and old rats increased on the first day after scalding, and then it would decrease. Furthermore, because CCR2 can promote the recruitment of macrophages at the inflammation site during tissue injury, it indirectly reflects the infiltration of macrophages at the injury site. (Fig. 3) However, we detected by ELISA that the CCL2 
concentration in the skin tissue of all rats increased compared to the control group on the first day after scalding and then decreased, but it was still higher than the control group. Furthermore, because CCL2 can promote the formation of M2 macrophages, the increased M2 macrophages can promote tissue repair. (Fig. 2)

We also used IHC staining and HE staining to observe the infiltrated cells. CD +68 cells are the most reliable markers of macrophages. In the HE stained cell smear, the infiltration of many inflammatory cells can observe in the skin tissues on the 7th day after scalding. It proves that the scald causes an inflammatory response in rat skin tissues. (Fig. 6) In the IHC stained smear, we can observe the infiltration of a large number of CD68 + cells. Therefore, it is proving that macrophages are involved in the inflammatory response of scalded skin tissues. (Fig. 5)

\section{Discussion}

Our experiment used the skin of rats to construct an inflammation model after scalding. Scald is a common form of injury. Local inflammation in tissue damage is essential for wound healing and the defense against infection. ${ }^{[16]}$ Macrophages run through all stages of the repair response, and they increase in the inflammation stage, reach a peak in the tissue formation stage, and gradually decrease in the mature stage. ${ }^{[17]}$ The early stage of the repair reaction is mainly inflammation, which locally activates the innate immune system, initiates a local inflammatory response, and recruits inflammatory cells from the blood circulation. ${ }^{[18,19]}$ The recruited macrophages are activated by proinflammatory mediators released by the serum after injury, leading to the expression of VEGF and TGF- $\beta 1$ and inducing the proliferation of fibroblasts and endothelial cells and contributing to wound angiogenesis and myofibroblast differentiation. ${ }^{[20]}$ The repair response enters the late stage, mainly anti-inflammatory, cell proliferation is weakening, the production and release of mononuclear macrophages reduced, and the expression of VEGF and TGF-b1 continues to decline, which ultimately weakens the degree of granulation tissue formation. ${ }^{[21]}$

Tenascin- $C$ has been involved in angiogenesis as an extracellular signal molecule $\mathrm{e}^{[22]}$. VEGF and CCL2 secretion in the supernatant was determined by ELISA assay. The data revealed that tenascin-c markedly prompted VEGF and CCL2 secretion and mRNA expression in macrophages. Therefore, we found tenascin-c could confer a proangiogenic effect to macrophages. It shows that TNC is not only involved in inflammation but also tissue repair possibly. (Fig. 2)

On the first day after scald, compared with the control group, the serum TN-C and VEGF concentrations of young rats increased significantly. However, the serum concentrations in aged rats decreased. This may be related to the causes of "inflamm-aging". Although there is no exact understanding about it. However, several common molecular pathways such as autophagy have been identified that seem to be associated with both aging and lowgrade inflammation. Autophagy, the cell machinery process that removes damaged proteins and large aggregates, is also slowed up at older age and in age-related disease, causing damaged material to accumulate and reduce cellular efficiency. The down-regulation of the 
related expression of TNC mRNA and VEGF mRNA in aged rats indicates that Tenascin-C protein is cleared from the tissues, and it is unlikely to play a role in long-term ECM remodeling.

The study found that the content of TNC increased in the inflammation site, and its mRNA expression decreased during the late stage of the scald. TNC can combine with cell surface receptors and further directly affect cell signal transduction and cell proliferation. ${ }^{[5-7]}$ In mouse synovial macrophages, TNCinduced integrin-mediated signal transduction up-regulates various proinflammatory molecules, including CCL2 ${ }^{[8]}$ The differential regulation of the chemokine system integrates polarized macrophages in the pathway of tissue repair and remodeling. CCL2 is a chemokine related to the polarization of CD $4+$ Th2. CCR2 is necessary for the recruitment of macrophages to tissues during injury. When proinflammatory signals spread throughout the body, they may down-regulate the expression of inflammatory chemokine receptors such as CCR2 to provide a stop signal to recruited macrophages to inhibit excessive macrophage recruitment at sites of inflammation and tissue damage.

\section{Conclusion}

We can reasonably speculate that macrophage migration was prompted by tenascin-c exposure. And TNC promotes skin inflammation and healing after scalding by inducing the infiltration of macrophages. This may be related to the expression of annexin II. Some studies have shown that Annexin II acts as a cell surface receptor of tenascin-c and was demonstrated to mediate the effect of tenascin-c on macrophage migration ${ }^{[23]}$. Modulation of tissue levels of humoral factors such as VEGFA, regulation of cell response to key growth factors, and direct effects on cell morphology and adhesion are mechanisms used by tenascin- $C$ to guide blood vessel formation during repair ${ }^{[4]}$.

This experiment mainly explored the relationship between macrophages and tenascin $\mathrm{C}$, and did not detect the influence of neutrophils on them. In addition, we only use CD68 + to label macrophages. Therefore, in further research, we will use more antibodies to mark macrophages and study the relationship between neutrophils, macrophages and tenascin C. At present, we can only conclude that TNC promotes inflammation and wound healing, which is also accompanying angiogenesis, and the infiltration of macrophages leads to the occurrence of inflammation. Regarding inflammation-aging, the focus of research on improving the understanding of aging and age-related disease inflammation regression mechanisms should also be given priority, since this is an under-researched area. All these molecular pathways are, or have the potential to be developed as drug targets for clinical intervention, help to alleviate and regulate inflammation, and may play a role in delaying the onset or treatment of agerelated diseases.

\section{Declarations}

\section{Funding}


This work was supported by grants from President Foundation of Nanfang Hospital, Southern Medical University (2019C032 to Kai Qian) and Natural Science Foundation of Guangdong Province, China (2018A0303130312 to Hao Liu).

\section{Competing Interests}

The authors have no relevant financial or non-financial interests to disclose.

\section{Authors' contributions}

Hao Liu contributed to the study's conception and design. Material preparation, data collection and analysis were performed by Kai Qian and Hao Liu. All the research was performed together by Weiquan Zhong $\square$ Jin Wang Wenjun Liu and Yixin Mao. The first draft of the manuscript was written by Weiquan Zhong and all authors commented on previous versions of the manuscript. All authors read and approved the final manuscript.

\section{Ethics approval}

This study was performed in line with the principles of the Declaration of Helsinki. All applicable international, national, and/or institutional guidelines for the care and use of animals were followed.The procedures for care and use of animals were approved by the Ethics Committee of the Southern Medical University and all applicable institutional and governmental regulations concerning the ethical use of animals were followed.

\section{Consent to participate and for publication}

All authors agrees to publication in the Journal indicated below and also to publication of the article in English by Springer in Springer's corresponding English-language journal. The copyright to the Englishlanguage article is transferred to Springer effective if and when the article is accepted for publication. The author warrants that his/her contribution is original and that he/she has full power to make this grant. All authors signs for and accepts responsibility

for releasing this material on behalf of any and all co-authors. The copyright transfer covers the exclusive right to reproduce and distribute the article, including reprints, translations, photographic, reproductions, microform, electronic form (offline, online) or any other reproductions of similar nature.

\section{Availability of data and material}

The datasets used or analysed during the current study are available from the corresponding author on reasonable request.All data generated or analysed during this study are included in this published article and its supplementary information files.

\section{Acknowledgments}


This study was supported by grants from the Guangdong Natural Science Foundation (2018A0303130312) and President Foundation of Nanfang Hospital, Southern Medical University (2019C032) .

\section{References}

1. Midwood, K.S., et al., Tenascin-C at a glance. J Cell Sci, 2016. 129(23): p.4321-4327.

2. Imanaka-Yoshida, K., T. Yoshida, and S. Miyagawa-Tomita, Tenascin-C in development and disease of blood vessels. Anat Rec (Hoboken), 2014. 297(9): p. 1747-57.

3. Udalova, I.A., et al., Expression and immune function of tenascin-C. Crit Rev Immunol, 2011. 31(2): p. 115-45.

4. Midwood, K.S, and G. Orend, The role of tenascin-C in tissue injury and tumorigenesis. J Cell Commun Signal, 2009. 3(3-4): p. 287-310.

5. Midwood, K. et al., Tenascin-C is an endogenous activator of Toll-like receptor 4 that is essential for maintaining inflammation in arthritic joint disease. NatMed, 15(7): p. 774-80.

6. Huang, W., et al., Interference of tenascin-C with syndecan-4 binding to fibronectin blocks cell adhesion and stimulates tumor cell proliferation. Cancer Res, 2001. 61(23): p. 8586-94.

7. Midwood, K.S., et al., Coregulation of fibronectin signaling and matrix contraction by tenascin-C and syndecan-4. Mol Biol Cell, 2004. 15(12): p. 5670-7

8. Kanayama, M. et al., Alpha9 integrin and its ligands constitute critical joint microenvironments for development of autoimmune arthritis. J Immunol, 2009. 182(12): p. 8015-25.

9. Koh, T.J. and L.A. DiPietro, Inflammation and wound healing: the role of the macrophage. Expert Rev Mol Med, 2011. 13: p. e23.

10. Kovacs, E.J. and L.A. DiPietro, Fibrogenic cytokines and connective tissue production. FASEB J, 1994. 8(11): p. 854-61.

11. Leibovich, S.J. and R. Ross, The role of the macrophage in wound repair. A study with hydrocortisone and antimacrophage serum. Am J Pathol, 1975. 78(1): 71-100.

12. Polverini, P.J. et al., Activated macrophages induce vascular proliferation. Nature, 1977. 269(5631): p. 804-6.

13. Murray, P.J., Macrophage Polarization. Annu Rev Physiol, 2017. 79: p. 541-566.

14. Diegelmann, R.F., and M.C. Evans Wound healing: an overview of acute, fibrotic and delayed healing. Front Biosci, 2004. 9: p. 283-9.

15. Martinez, F.O., L. Helming, and S. Gordon, Alternative activation of macrophages: an immunologic functional perspective. Annu Rev Immunol, 2009. 27: p. 451-83.

16. Church, D., et al., Burn wound infections. Clin Microbiol Rev, 2006. 19(2): p. 403-34.

17. Martin, P. and S.J. Leibovich, Inflammatory cells during wound repair: the good, the bad and the ugly. Trends Cell Biol, 2005. 15(11): p. 599-607. 
18. Werner, S., T. Krieg, and H. Smola, Keratinocyte-fibroblast interactions in wound healing. J Invest Dermatol, 2007. 127(5): p. 998-1008.

19. Babcock, D.T., et al., Circulating blood cells function as a surveillance system for damaged tissue in Drosophila larvae. Proc Natl Acad Sci U S A, 2008. 105(29): p. 10017-22.

20. Zhang, X. and D.M. Mosser, Macrophage activation by endogenous danger signals. J Pathol, 2008. 214(2): p. 161-78.

21. Lucas, T., et al., Differential roles of macrophages in diverse phases of skin repair. J Immunol, 2010. 184(7): p. 3964-77.

22. Tanaka $\mathrm{K}$, Hiraiwa $\mathrm{N}$, Hashimoto $\mathrm{H}$,et al. Tenascin-C regulates angiogenesis in tumor through the regulation of vascular endothe-lial growth factor expression. Int J Cancer.2004; 108: 31-40.

23. Wang Z, Wei Q, Han L, Cao K, Lan T, Xu Z, Wang Y, Gao Y, Xue J, Shan F, Feng J, Xie X. Tenascin-c renders a proangiogenic phenotype in macrophage via annexin II. J Cell Mol Med. 2018 Jan;22(1):429-438. doi: 10.1111/jcmm.13332. Epub 2017 Aug 30. PMID: 28857429; PMCID: PMC5742692.

24. Swindle CS, Tran KT, Johnson TD, Banerjee P, Mayes AM, Griffith L, Wells A. Epidermal growth factor (EGF)-like repeats of human tenascin-C as ligands for EGF receptor. J Cell Biol. $2001 \mathrm{Jul}$ 23;154(2):459-68. doi: 10.1083/jcb.200103103. PMID: 11470832; PMCID: PMC2150768.

\section{Figures}

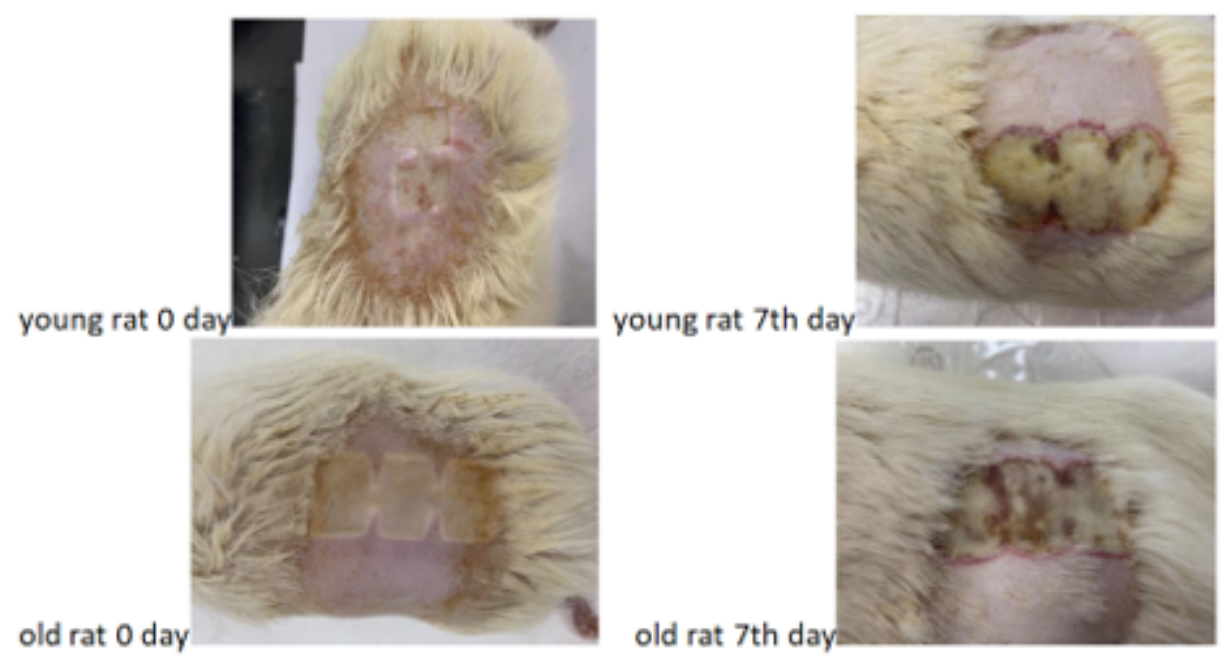

\section{Figure 1}

See image above for figure legend. 

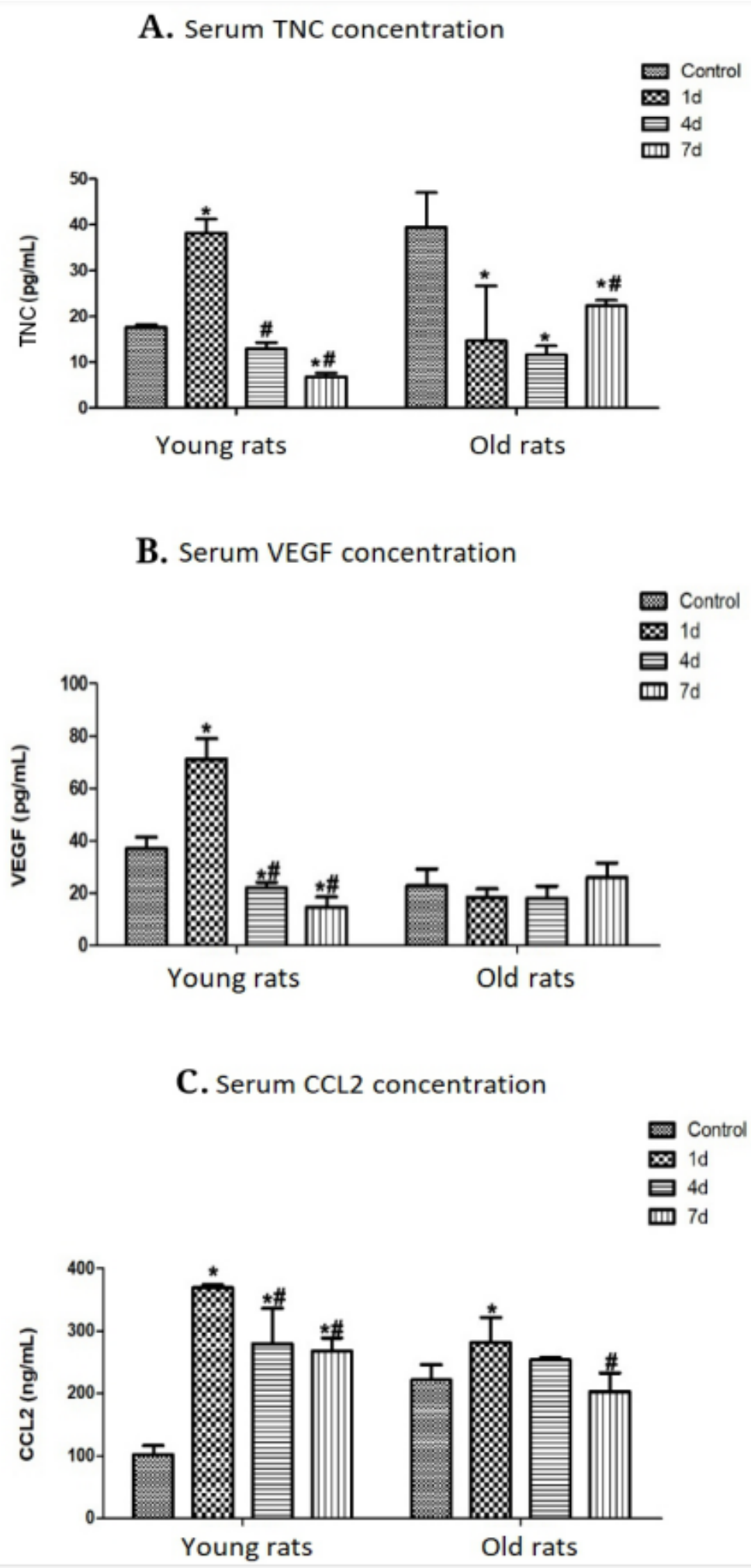

Figure 2

See image above for figure legend. 
D. Relative expression quantity of TNC mRNA

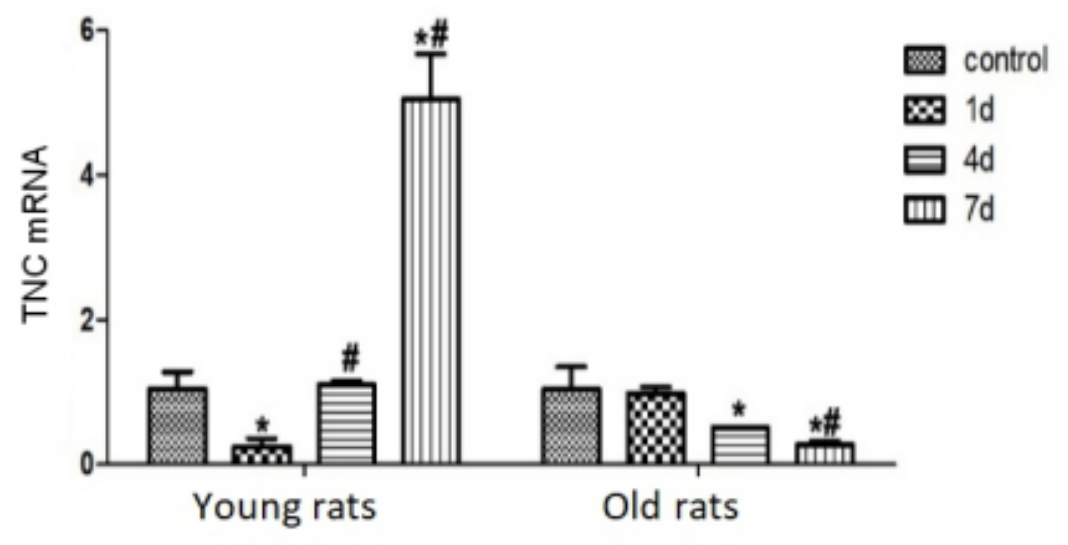

E. Relative expression quantity of VEGF MRNA

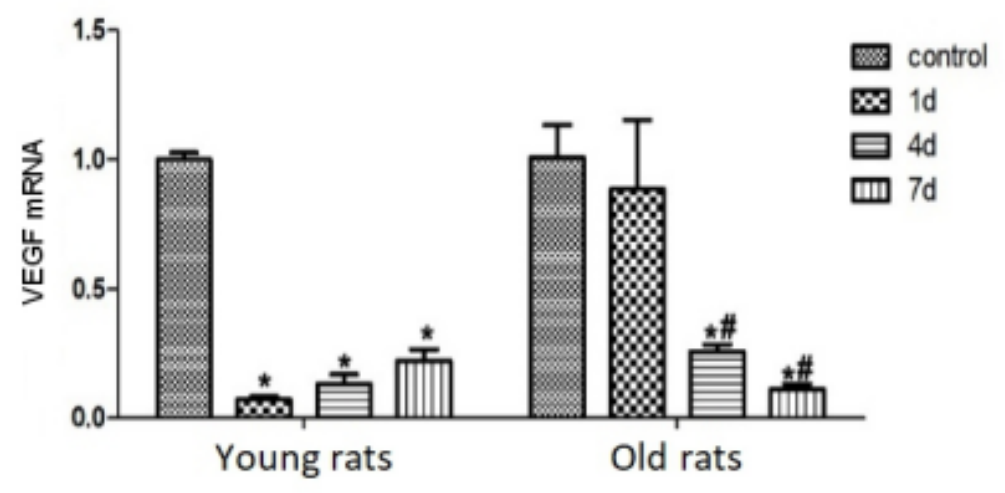

F. Relative expression quantity of CCR2 mRNA

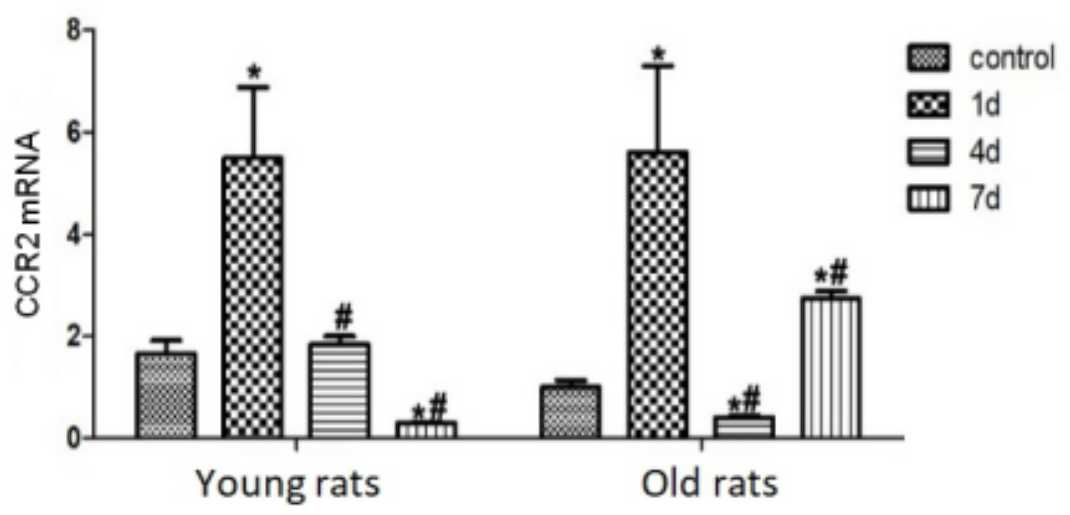

Figure 3

See image above for figure legend. 
G.Detection of the expression quantity of TNC, VEGFA and CCR2 in skin

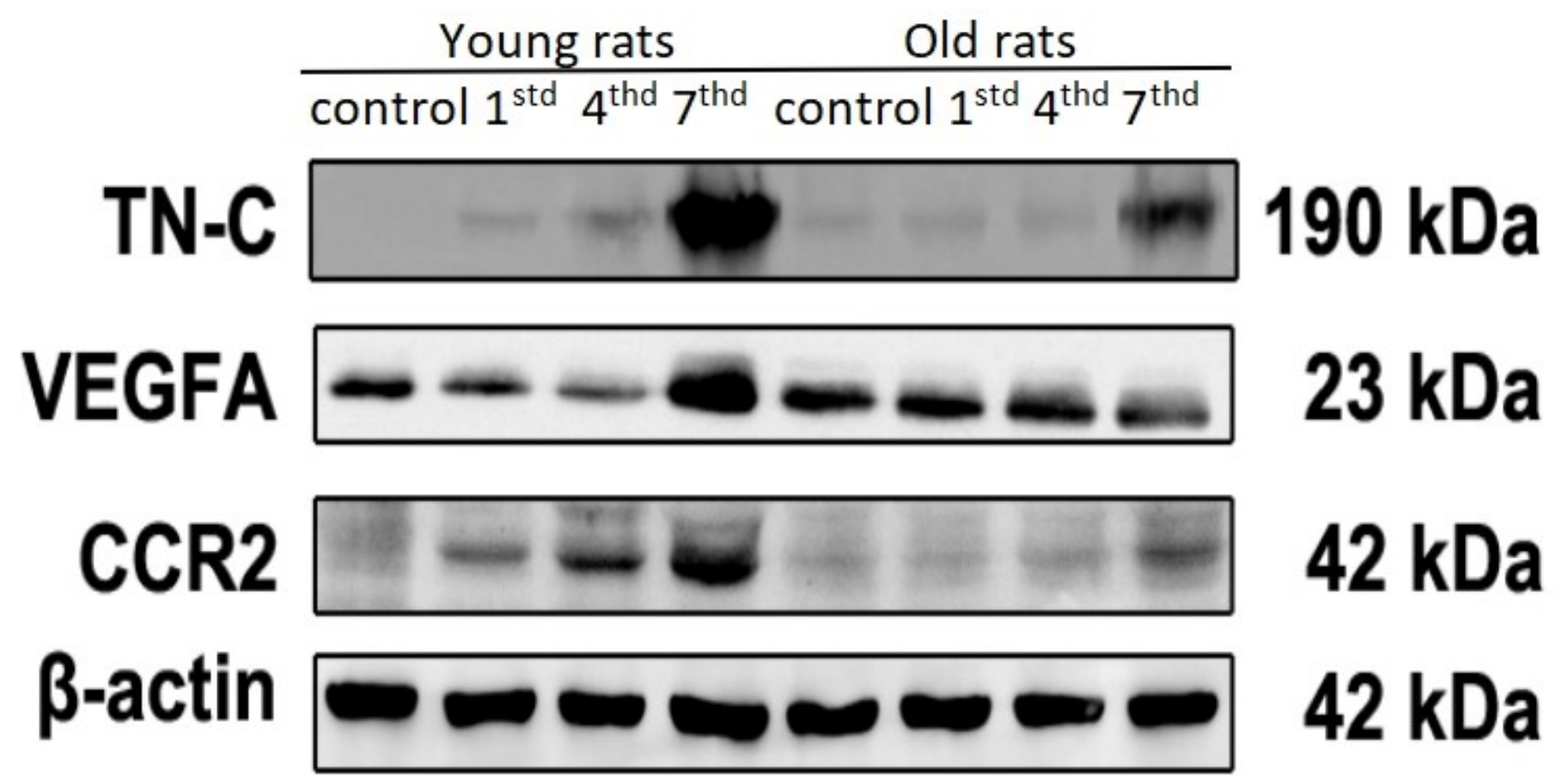

Figure 4

See image above for figure legend.

Figure 5

See image above for figure legend.

Figure 6

See image above for figure legend. 